\title{
Angiotensin-converting enzyme insertion/deletion polymorphism is a risk factor for thoracic aortic aneurysm in patients with bicuspid or tricuspid aortic valves
}

\author{
Ilenia Foffa, MSc, ${ }^{\text {a,b }}$ Michele Murzi, MD, ${ }^{\text {b,c }}$ Massimiliano Mariani, MD, ${ }^{c}$ Anna Maria Mazzone, MD, ${ }^{c}$ \\ Mattia Glauber, MD, ${ }^{\mathrm{c}}$ Lamia Ait Ali, MD, PhD, ${ }^{\mathrm{a}}$ and Maria Grazia Andreassi, MSc, $\mathrm{PhD}^{\mathrm{a}, \mathrm{c}}$
}

\begin{abstract}
Objective: The angiotensin-converting enzyme (ACE) is highly expressed in the aneurysmal vascular wall, in both animal models and human disease. Genetic variations in ACE could be crucial in determining the risk of thoracic aortic aneurysm (TAA). The aim of the present study was to examine the role of ACE insertion/deletion polymorphism on the risk of TAA in patients with bicuspid aortic valves or tricuspid aortic valves.
\end{abstract}

\begin{abstract}
Methods: We enrolled 216 patients (158 men; age, $58.9 \pm 14.9$ years) with TAA, associated with bicuspid aortic valves $(\mathrm{n}=105)$ and tricuspid aortic valves $(\mathrm{n}=111)$ compared with 312 patients $(252 \mathrm{men}$; age, $54.6 \pm 11.0$ years) with angiographically proven coronary artery disease and 300 healthy controls ( 91 men; age, $40.4 \pm 10.5$ years).

Results: The genotype distribution of ACE insertion/deletion was significantly different between the patients with TAA compared with both the control group $(P=.0005)$ and the coronary artery disease group $(P=.03)$. The genotypes were not different between the control group and the coronary artery disease group $(P=.3)$. Compared with the controls, both the bicuspid aortic valve patients $(P=.0008)$ and tricuspid aortic valve patients $(P<.0001)$ had a greater frequency of allele $\mathrm{D}$. The aortic diameters were significantly different among the three genotypes $(48.3 \pm 6.6,45.3 \pm 8.9,39.9 \pm 8.7$ for the DD, DI, and II genotypes, respectively; $P=.0002)$. A synergistic effect between the ACE D allele and hypertension was found for both an increased aortic diameter $(P=.003)$ and the risk of TAA $(P<.001)$. On multivariate logistic regression analysis, D allele (odds ratio, 3.0; $95 \%$ confidence interval, $1.1-8.1 ; P=.03$ ) was a significant predictor of TAA.
\end{abstract}

Conclusions: ACE insertion/deletion polymorphism represents a genetic biomarker for TAA. These findings could have a significant effect on both the early detection and effective pharmacologic treatment of aortic disease. (J Thorac Cardiovasc Surg 2012;144:390-5)

Thoracic aortic aneurysm (TAA), involving the ascending aorta, is a highly fatal cardiovascular disorder. ${ }^{1}$ Aneurysms of the ascending thoracic aorta most often result from cystic medial degeneration, associated with tricuspid aortic valves (TAVs) or congenital bicuspid aortic valves (BAVs). Clinical studies have also shown that ascending aortic dilatation occurs more frequently and at a younger age in patients with BAVs than it does in patients with normal TAVs. ${ }^{1-3}$

During the past decade, several studies have investigated the genetic basis of TAA, focusing on its relation to systemic connective tissue disorders, such as Marfan syndrome

From the CNR Institute of Clinical Physiology, ${ }^{a}$ Pisa, Italy; Scuola Superiore Sant'Anna, ${ }^{\text {b }}$ Pisa, Italy; and G. Monasterio Foundation CNR-Regione Toscana, Massa, Italy.

This research was partially funded by Ente Cassa di Risparmio di Firenze.

Disclosures: Authors have nothing to disclose with regard to commercial support.

Received for publication Feb 24, 2011; revisions received Nov 23, 2011; accepted for publication Dec 14, 2011; available ahead of print Jan 16, 2012.

Address for reprints: Maria Grazia Andreassi, MSc, PhD, CNR Institute of Clinical Physiology, G. Pasquinucci Hospital, Via Aurelia Sud-Montepepe, Massa 54100 Italy (E-mail: andreas@ifc.cnr.it).

$0022-5223 / \$ 36.00$

Copyright (C) 2012 by The American Association for Thoracic Surgery doi:10.1016/j.jtcvs.2011.12.038 and Ehlers-Danlos syndrome, and/or familial cases with an autosomal dominant pattern. ${ }^{1-4}$

Several mutations and loci have been identified, and additional studies to identify the specific genes involved in the pathogenesis of familial but nonsyndromic TAAs are in progress. ${ }^{4,5}$

Common genetic variants could also play a role in the occurrence and progression of TAA, but they are largely unknown. ${ }^{6}$

The identification of the biochemical and genetic biomarkers is a fundamental strategy to monitor the development and progression of the aortic diameter and to tailor the medical and surgical approaches to this serious condition. ${ }^{2,6}$

The renin-angiotensin system (RAS) has been suggested to be crucial in the development of both abdominal aortic aneurysms (AAAs) and TAAs. ${ }^{7}$ Genetic variability in the RAS might, thus, play a role in the development of TAAs, allowing individualized treatment of patients at risk of acute aortic syndromes. Recent studies have shown that the angiotensin-converting enzyme (ACE) insertion/deletion (I/D) polymorphism represents a marker of increased susceptibility to AAAs. ${ }^{8-10}$ The aim of the present study was 

Abbreviations and Acronyms
$\mathrm{AAA}=$ abdominal aortic aneurysm
$\mathrm{ACE}=$ angiotensin-converting enzyme
$\mathrm{BAV}=$ bicuspid aortic valve
$\mathrm{CAD}=$ coronary artery disease
$\mathrm{CI}=$ confidence interval
$\mathrm{I} / \mathrm{D}=$ insertion/deletion
$\mathrm{OR}=$ odds ratio
RAS $=$ renin-angiotensin system
TAA $=$ thoracic aortic aneurysm
$\mathrm{TAV}=$ tricuspid aortic valve

to examine the role of ACE I/D polymorphism in the development of TAAs in patients with BAVs and TAVs.

\section{METHODS \\ Subjects}

From January 2008 to March 2010, we enrolled 216 patients (158 men; age $58.9 \pm 14.9$ years) who were referred to our cardiac surgery department for TAA, associated with BAVs $(n=105)$ or TAVs $(n=111)$.

Of these 216 patients, 90 had limited dilatation (maximal aortic diameter $<45 \mathrm{~mm}$ ), and 126 had a large aneurysm (aortic diameter $\geq 45 \mathrm{~mm}$ ), estimated using computed tomography scans and/or angiography. Of the patients with an aneurysm (aortic diameter $\geq 45 \mathrm{~mm}) 53(42 \%)$ and 73 $(58 \%)$ had BAVs or TAVs, respectively. A total of 167 patients underwent elective surgery for replacement of the ascending aorta and/or aortic valve replacement.

We also included a group of 312 patients ( 252 men; age, $54.6 \pm 11.0$ years) with angiographically proven coronary artery disease (CAD).

A group of 300 healthy subjects ( $91 \mathrm{men}$; age, $40.4 \pm 10.5$ years), who were members of the medical and technical staff of our institution, and who were negative for any clinical evidence of cardiovascular disease, was used as the control group.

A complete history, including cardiovascular risk factors, such as hypertension, diabetes, and dyslipidemia, was collected from all patients. The following definitions were used for the risk factors: hypertension, blood pressure $\geq 140 / 90 \mathrm{~mm} \mathrm{Hg}$ (confirmed by measurements on several occasions), or antihypertensive therapy; and dyslipidemia, low-density lipoprotein $130 \mathrm{mg} / \mathrm{dL}$ or more, high-density lipoprotein less than $35 \mathrm{mg} / \mathrm{dL}$, triglycerides $200 \mathrm{mg} / \mathrm{dL}$ or more, or the use of lipid-lowering medications. Current smokers were defined as patients with moderate or heavy use of cigarettes ( $\geq 3$ cigarettes/day) at entry into the study. Former smokers were defined as the participants who had been smoking regularly, at least 3 cigarettes/day and who had quit smoking for at least 6 months at enrollment. Nonsmokers were defined as patients who had never smoked before admission. The smoker group combined the past and current smokers. Accordingly, each risk factor was coded as either present or absent. Those with Marfan syndrome and traumatic aneurysms were excluded from the present study.

The aortic diameters were measured by imaging only in the patients referred to our cardiac surgery department for TAA. All participants provided informed consent, and the local ethical research committee approved the study.

\section{Detection of ACE Polymorphism}

Genotyping analysis was performed by our laboratory staff, who were unaware of the clinical data. Genomic DNA was extracted from the peripheral blood leukocytes.
The ACE I/D polymorphism was genotyped as previously described in published protocols. ${ }^{8-10}$ In brief, polymerase chain reaction was performed with 10 pmol of each primer: sense oligo $5^{\prime}$-CTG GAG ACC ACT CCC ATC CTT TCT- $3^{\prime}$ and anti-sense oligo $5^{\prime}$-GAT GTG GCC ATC ACA TTC GTC AGA T- $3^{\prime}$ in a final volume of $50 \mu \mathrm{L}$, containing $3 \mathrm{mM}$ of magnesium chloride, $50 \mathrm{mM}$ of potassium chloride, $10 \mathrm{mM}$ of Tris (hydroxymethyl) aminomethane hydrochloride ( $\mathrm{pH} 8.4), 0.1 \mathrm{mg} / \mathrm{mL}$ of gelatin, 0.5 $\mathrm{mM}$ of deoxynucleotidetriphosphate, and 1 unit of Taq polymerase. The DNA was amplified for 30 cycles with denaturation at $94^{\circ} \mathrm{C}$ for 1 minute, annealing at $58^{\circ} \mathrm{C}$ for 1 minute, and extension at $72^{\circ} \mathrm{C}$ for 2 minutes. The ACE I/D polymorphism consists of the presence/absence of a 287-bp DNA fragment corresponding to an Ala repetitive sequence in the intron 16 of the ACE gene, determining the insertion (I) and deletion (D) allele.

The polymerase chain reaction products corresponding to the insertion (480 bp) or deletion (193 bp) allele were analyzed on 1.5\% agarose gel stained with ethidium bromide.

\section{Statistical Analysis}

Statistical analyses of the data were performed with the StatView statistical package, version 5.0.1 (Abacus Concepts, Berkeley, Calif).

Data are expressed as the mean \pm standard deviation. Differences in noncontinuous variables were tested by chi-square analysis. Differences between the mean of the 2 continuous variables were evaluated using Student $t$ test. The data for three different groups were analyzed using analysis of variance, and significant differences among pairs of the mean values were tested using Scheffe's test.

The Scheffe test was chosen for multiple comparisons, because it is very robust for the violations of the assumptions that are typically associated with multiple comparisons procedures. Interaction terms were also introduced in the model to examine the interaction of the genotypes with hypertension. We calculated the odds ratios (ORs) and $95 \%$ confidence intervals (CIs) for the association of the ACE I/D polymorphism with the presence of TAA using unconditional logistic regression analysis. Multivariate logistic regression analysis was used to estimate the ORs after adjustment for the other vascular risk factors. Power calculations showed that the study sample was able to detect an association between ACE I/D polymorphism and TAA with ORs of 2.5 to 3.0, based on a deletion allele frequency of $0.6{ }^{6}$

\section{RESULTS}

The demographic and clinical characteristics of the study population are reported in Table 1 . Age $(P<.0001)$ and the proportion of men $(P<.0001)$ were significantly greater in the TAA group than in the control group. The prevalence of smoking $(P=.04)$, hypertension $(P<.0001)$, and dyslipidemia $(P<.0001)$ was also significantly greater in the TAA and CAD patients than in the control subjects (Table 1). The prevalence of atherogenic risk factors (including age, gender, hypertension, diabetes, cigarette smoking, dyslipidemia) was also significantly greater in the CAD group than in the control group (Table 1$)$. Age $(P=.0002)$ and the incidence of hypertension $(P=.04)$ were significantly greater in the TAA group than in the CAD group (Table 1).

The ACE I/D genotype distribution of the study populations is summarized in Table 2. The genotype distribution of ACE I/D was significantly different between the patients with TAA and both the control $(P=.0005)$ and the CAD $(P=.03)$ group. No significant difference was found in the genotype frequency between the control group and the CAD group $(P=.3)$. 
TABLE 1. Clinical and demographic characteristics of study population

\begin{tabular}{lccc}
\hline Characteristic & $\begin{array}{c}\text { TAA group } \\
(\mathbf{n}=\mathbf{2 1 6})\end{array}$ & $\begin{array}{c}\text { CAD group } \\
(\mathbf{n = 3 1 2})\end{array}$ & $\begin{array}{c}\text { Control group } \\
(\mathbf{n}=\mathbf{3 0 0})\end{array}$ \\
\hline Mean age & $58.9 \pm 14.9^{*}, \dagger$ & $54.6 \pm 11.0^{*}$ & $40.4 \pm 10.5$ \\
Male gender & $158(73.1)^{*}, \dagger$ & $252(80.8)^{*}$ & $91(30.3)$ \\
Smoking habit & $89(41.2) \S, \|$ & $203(65.1)^{*}$ & $95(31.7)$ \\
Diabetes mellitus & $14(6.5)$ & $31(9.9) \S$ & $10(3.3)$ \\
Dyslipidemia & $63(29.2)^{*}, \|$ & $230(73.7)^{*}$ & $27(9.0)$ \\
Hypertension & $112(51.8)^{*}, \ddagger$ & $134(42.9)^{*}$ & $29(9.7)$ \\
\hline
\end{tabular}

Date presented as mean \pm standard deviation or numbers, with percentages in parentheses. TAA, Thoracic aortic aneurysm; $C A D$, coronary artery disease. $* P<.0001$ vs controls. $\dagger P<.01$ vs CAD. $\ddagger P<.05$ vs CAD. $\S P<.05$ vs controls. $\| P<.0001$ vs CAD.

The ACE deletion allele was significantly more frequent in both BAV $(64 \%, P=.0008)$ and TAV $(64 \%, P<.0001)$ patients than in the control group (57\%).

When patients were stratified by the presence of a large aneurysm (aortic diameter $\geq 45 \mathrm{~mm}$ ), the ACE deletion allele was still significantly more frequent in both $\operatorname{BAV}(71 \%$, $P=.03)$ and TAV $(71 \%, P=.001)$ patients than in the control group $(57 \%)$.

Univariate logistic regression analysis demonstrated that ACE I/D polymorphism was related to nearly a threefold increased TAA risk in both the dominant and the additive genetic models (Table 3 ).

The aortic diameter was also significantly different among the three genotypes $(48.3 \pm 6.6,45.3 \pm 8.9$, and $39.9 \pm 8.7$ for the DD, DI, and II genotypes, respectively; $P=.0002)$. Patients with the DD $(P=.0003)$ and ID $(P=.003)$ genotypes had significantly greater aortic diameters than patients with the II genotype. Also, a significant difference $(P=.04)$ was found in the DD versus ID patients (Figure 1).

The aortic diameters were also significantly different among the three genotypes for both BAV and TAV patients (Figure 1). Furthermore, we found a synergistic effect between the ACE deletion allele and hypertension (Figure 2) for both increased aortic diameter $(P=.03)$ and the risk of TAA $(P=.001)$. On multivariate logistic regression analysis, hypertension (OR, 1.7; 95\% CI, 1.0-6.1; $P=.04)$, age (OR, $1.0 ; 95 \% \mathrm{CI}, 1.0-1.1 ; P=.03)$, male

TABLE 2. Genotype distribution of ACE I/D polymorphism

\begin{tabular}{lcccc}
\hline Genotype & $\begin{array}{c}\text { Allele } \\
\text { frequency }\end{array}$ & $\begin{array}{c}\text { Control } \\
\text { group }\end{array}$ & $\begin{array}{c}\text { CAD } \\
\text { group }\end{array}$ & $\begin{array}{c}\text { TAA } \\
\text { group*, } \dagger\end{array}$ \\
\hline ACE II & & $65(21.7)$ & $54(17.3)$ & $20(9.3)$ \\
ACE ID & & $126(42.0)$ & $144(46.2)$ & $115(53.2)$ \\
ACE DD & & $109(36.3)$ & $114(36.5)$ & $81(37.5)$ \\
& ACE D & $0.57 \%$ & $0.60 \%$ & $0.64 \% \ddagger, \S$ \\
\hline
\end{tabular}

Data presented as numbers, with percentages in parentheses, unless otherwise noted. $A C E$, Angiotensin-converting enzyme; $I / D$, insertion/deletion; other abbreviations as in Table $1 .{ }^{*} P<.01$ vs controls. $\uparrow P<.05$ vs CAD. $\uparrow_{\uparrow} P<.0001$ vs controls. $\S P<.01$ vs controls.
TABLE 3. Association between ACE I/D polymorphism and ascending aortic aneurysm

\begin{tabular}{lcc}
\hline \multicolumn{1}{c}{ Model } & OR $(\mathbf{9 5} \% \mathbf{C I})$ & $\boldsymbol{P}$ value \\
\hline Additive (DD vs DI vs II) & $2.7(1.5-5.2)$ & .001 \\
Dominant (DD and ID vs II) & $2.8(1.6-5.0)$ & .0004 \\
Recessive (DD versus ID and II) & $1.2(0.8-1.8)$ & .3 \\
\hline$O R$, Odds ratio; $C I$, confidence interval; other abbreviations as in Table 2.
\end{tabular}

gender (OR, 2.6; 95\% CI, 1.2-5.6; $P=.02$ ), and deletion allele (OR, 3.0; 95\% CI, 1.1-8.1; $P=.03$ ) were significant determinants of TAA.

\section{DISCUSSION}

The present study showed that the ACE deletion allele is significantly associated with both a large aortic diameter and an increased risk of TAA. In addition, we observed a synergic effect between the ACE I/D polymorphism and hypertension on the risk of TAA.

\section{RAS, ACE I/D Polymorphism, and Aortic Aneurysms}

Increasing data have consistently reported that inappropriate activity of the RAS is an underlying cause of aortic aneurysmal disease. ${ }^{7}$ For instance, infusion of angiotensin II in apolipoprotein E-deficient mice induced large aneurysm formation independently of blood pressure changes. ${ }^{11,12}$ In addition, administration of losartan, the angiotensin II type 1 antagonist, completely attenuated the aortic pathology in a Marfan mouse model. ${ }^{13}$

Angiotensin II is known to play a crucial role in the remodeling of vascular tissues by activation of growth factors and cytokines, in addition to the regulation of blood pressure. $^{7,14}$

Angiotensin II also increases transforming growth factor$\beta$ transcription and synthesis and induces an increase in matrix metalloproteinases activity, leading to inflammation, extracellular matrix degradation, and apoptosis.,15-17 ACE plays a key role in the RAS, because it generates the active octapeptide angiotensin II from the decapeptide angiotension I.

Importantly, ACE is highly expressed in the aneurysmal vascular wall, both in human disease and animal models. ${ }^{18,19}$ In aneurysmal aortic specimens obtained during operative repair of patients, ACE activity was increased sixfold. ${ }^{19}$

It is well-known that patients with the DD genotype have high ACE levels in their plasma and cardiac tissue. ${ }^{20,21}$ Therefore, the very common ACE I/D polymorphism could contribute to the risk of aortic aneurysmal diseases. Recent studies have documented that ACE I/D polymorphism represents a susceptibility factor for AAA. ${ }^{8-10}$ Moreover, a large study of three geographically distinct, but ethnically similar, case-control cohorts confirmed a role for the $A C E$ gene in modulating the 

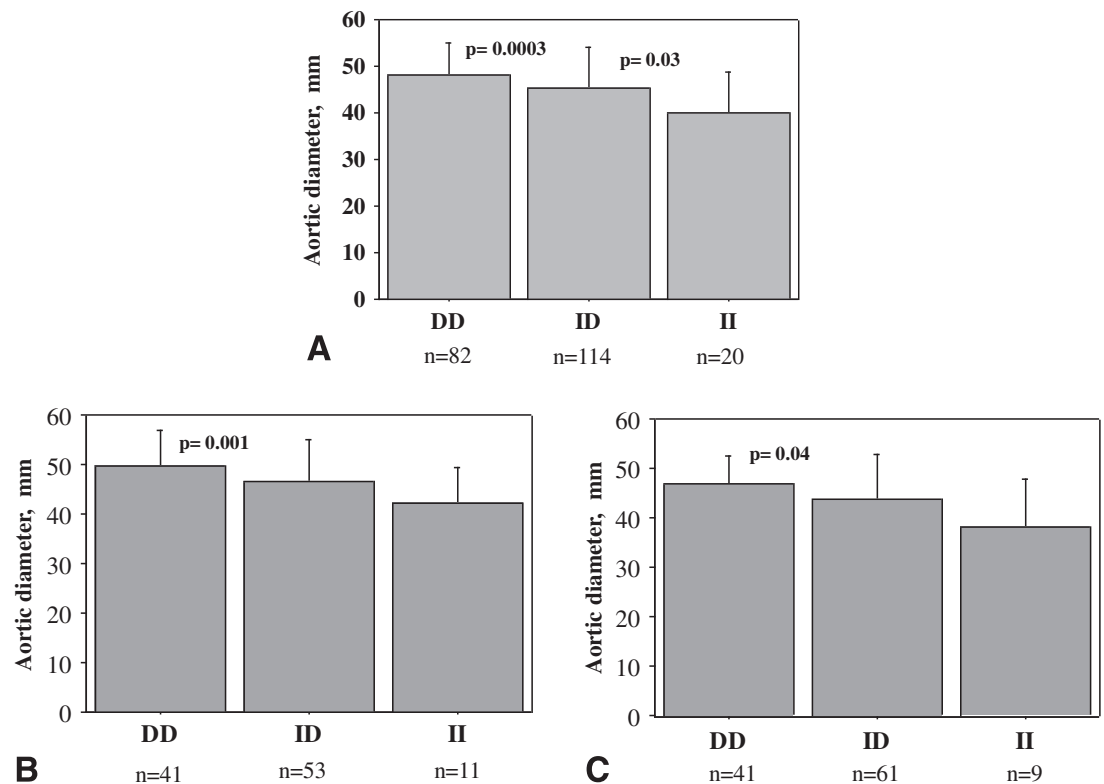

FIGURE 1. Aortic diameters according to angiotensin-converting enzyme insertion/deletion polymorphism for (A) all patients, (B) bicuspid aortic valve patients, and (C) tricuspid aortic valve patients.

susceptibility to the disease and demonstrated that the ATIR $1166 \mathrm{~A}>\mathrm{C}$ polymorphism represented a risk factor for $\mathrm{AAA}^{22}$

\section{Clinical and Pharmacogenetics Implications}

Our findings have relevant implications for the care of patients and research both. Current American College of Cardiology/American Heart Association guidelines recommend replacement of the ascending aorta in select patients (eg, patients with Marfan syndrome, BAV patients requiring concomitant aortic valve replacement) with a ascending aortic diameter greater than $4.5 \mathrm{~cm} .{ }^{23}$ These recommendations, however, were based almost exclusively on "expert opinion," and improved clinical predictors are needed to identify those at risk of acute aortic dissection and to determine the aortic diameter that justifies the risk of surgical repair of a TAA. ${ }^{24,25}$ Clinical studies of familial TAA have provided the evidence that the timing of surgical repair can be dictated by the underlying mutated gene causing the disease. ${ }^{24}$ In the clinical setting, the identification of common genetic risk factors for TAA could also facilitate risk stratification to prevent or attenuate the disease. ${ }^{2,6}$ The combination of genetic tests and clinical routine examinations could contribute to the personalized prevention of this condition. A better understanding of the genetic component predisposing to TAA remains the challenge for the future for selecting the optimal medical regimen for each patient. Currently, the medical therapies for aortic disease entail aggressive management of cardiovascular risk, especially hypertension, to control the growth of the aortic aneurysm and reduce its risk of dissection or rupture. Our findings suggest that RAS inhibition could be considered as first-line therapy in preventing aortic aneurysmal disease.

Furthermore, data from animal models have suggested that ACE inhibitors slow the progressive course of aortic
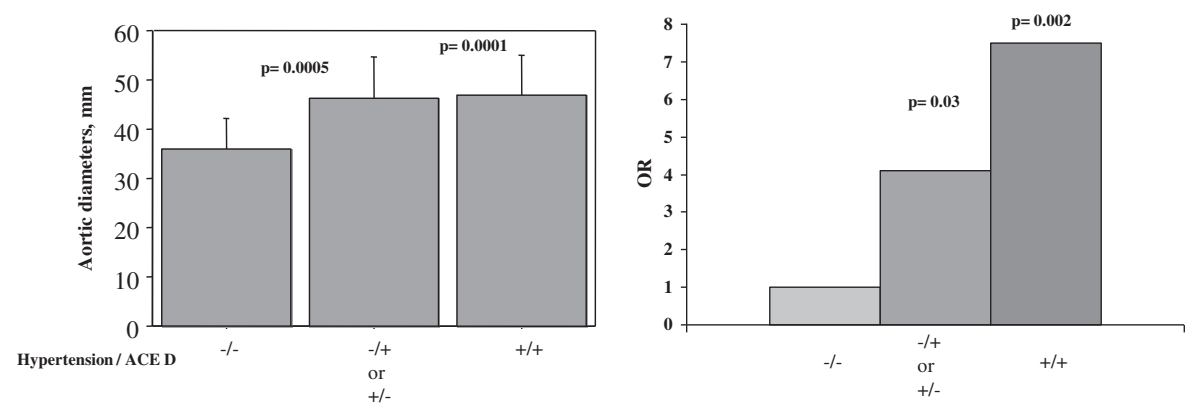

FIGURE 2. Interaction between angiotension-converting enzyme deletion allele and hypertension for aortic diameter and thoracic aortic aneurysm risk. 
aneurysms, and they are more effective at preventing rupture than other antihypertensive agents. ${ }^{13,26-28}$

A very recent experimental study has shown that the AT1 receptor blocker, losartan, which uniquely blocks transforming growth factor- $\beta$ activity, allowing intact AT2 signaling, abrogated aneurysm progression in the Marfan mice. In contrast, the ACE inhibitor, enalapril, which limits signaling through both receptors, was less effective. In the mouse studies, losartan reduced ERK1/2 phosphorylation through a combination of inhibiting AT1 receptormediated ERK activation and by shunting angiotensin II signaling through the AT2 receptor. This lends further support to the idea that an AT1 receptor blocker might be superior to ACE inhibitors for clinical treatment. ${ }^{29}$

ACE inhibitors have recently been associated with a reduced risk of rupture in patients with AAAs. ${ }^{30}$ Limited data, however, are currently available regarding the potential benefit of ACE inhibitors or angiotensin II receptor blockers for ascending aortic aneurysms.

Recent studies have demonstrated that ACE inhibitors significantly reduced both the aortic stiffness index and aortic root growth in patients with Marfan syndrome. ${ }^{31,32}$ The angiotensin II receptor blocker losartan has also been shown to reduce the rate of aortic dilatation in a small pediatric cohort of patients with Marfan syndrome. ${ }^{33}$

However, no evidence has suggested whether angiotensin II receptor blockers are preferable to $\beta$-blockers for aortic disease.

Currently, ongoing randomized clinical trials are comparing the efficacy of standard treatment with $\beta$-adrenoceptor blockade, with the AT1 receptor antagonism using losartan, in patients with Marfan syndrome. ${ }^{34-36}$ The results of these trials may help to better define which of these therapies is of greater benefit for the long-term treatment of these patients and to better clarify the role of the RAS in ascending aortic aneurysm.

However, genetic variants in the RAS genes could also modify the efficacy of the therapies. Therefore, it would be important to perform large pharmacogenetic studies to evaluate whether gene-drug interactions vary the risk of TAA and to identify specific populations that would benefit from specific treatments.

\section{Study Limitations}

Some important limitations must be considered when interpreting the results of the present study. First, the pathogenesis of aortic aneurysmal disease is undoubtedly complex, involving many genes acting in an additive or interactive manner, together with environmental factors. The RAS represents a perfect example in which cumulative variations of each component of this enzymatic cascade could strongly affect the overall activity of the system. ${ }^{37}$

An important limitation of our study was the lack of a more comprehensive genetic analysis of other RAS candidate genes (eg, angiotensinogen and angiotensin II type I receptor genes) and for which a functional polymorphism exists.

Second, given the case-control design, potential confounding factors can never be completely excluded in an observational study. Another limitation of our study was the nature of the control population, which consisted of volunteers or healthy donors. We could not rule out the possibility that these participants might have had asymptomatic aortic pathologic features because thoracic aortic imaging was not conducted. However, we also included a group of patients with atherosclerosis as another control group to better determine whether ACE polymorphism is causally related to the presence of TAA.

In conclusion, our results strongly support that ACE I/D polymorphism represents a genetic biomarker of increased susceptibility to TAA, confirming the key role of the RAS as an underlying cause of aneurysmal aortic disease. These findings could have a significant effect for both early detection and effective pharmacologic treatment of aortic disease.

We thank Marco Borbotti e Daniele Ferri for their administrative support.

\section{References}

1. Isselbacher EM. Thoracic and abdominal aortic aneurysms. Circulation. 2005; 111:816-28.

2. Eagleton MJ. Molecular diagnoses and treatments-past, present, or future? Semin Vasc Surg. 2007;20:128-34.

3. El-Hamamsy I, Yacoub MH. Cellular and molecular mechanisms of thoracic aortic aneurysms. Nat Rev Cardiol. 2009;6:771-86.

4. Pannu H, Tran-Fadulu V, Milewicz DM. Genetic basis of thoracic aortic aneurysms and aortic dissections. Am J Med Genet Semin. 2005;139:10-6.

5. Eagle KA. Rationale and design of the national registry of genetically triggered thoracic aortic aneurysms and cardiovascular conditions (GenTAC). Am Heart J. 2009; 157:319-26.

6. Ruigrok YM, Elias R, Wijmenga C, Rinkel GJ. A comparison of genetic chromosomal loci for intracranial, thoracic aortic, and abdominal aortic aneurysms in search of common genetic risk factors. Cardiovasc Pathol. 2008;17:40-7.

7. Lu H, Rateri DL, Cassis LA, Daugherty A. The role of the renin-angiotensin system in aortic aneurysmal diseases. Curr Hypertens Rep. 2008;10:99-106.

8. Yeung JMC, Heeley M, Gray S, Lingam MK, Manning G, Nash JR, et al. Does the angiotensin-converting enzyme (ACE) gene polymorphism affect rate of abdominal aortic aneurysm expansion? Eur J Vasc Endovasc Surg. 2002;24:69-71.

9. Fatini C, Pratesi G, Sofi F, Gensini F, Sticchi E, Lari B, et al. ACE DD genotype: a predisposing factor for abdominal aortic aneurysm. Eur J Vasc Endovasc Surg. 2005;29:227-32.

10. Lucarini L, Sticchi E, Sofi F, Pratesi G, Pratesi C, Pulli R, et al. ACE and TGFBR1 genes interact in influencing the susceptibility to abdominal aortic aneurysm. Atherosclerosis. 2009;202:205-10.

11. Daugherty A, Manning MW, Cassis LA. Angiotensin II promotes atherosclerotic lesions and aneurysms in apolipoprotein E-deficient mice. J Clin Invest. 2000; 105:1605-12.

12. Cassis LA, Gupte M, Thayer S, Zhang X, Charnigo R, Howatt DA, et al. ANG II infusion promotes abdominal aortic aneurysms independent of increased blood pressure in hypercholesterolemic mice. Am J Physiol Heart Circ Physiol. 2009;296:H1660-5.

13. Habashi JP, Judge DP, Holm TM, Cohn RD, Loeys BL, Cooper TK, et al. Losartan, an AT1 antagonist, prevents aortic aneurysm in a mouse model of Marfan syndrome. Science. 2006;312:117-21.

14. Tsunemi K, Takai S, Nishimoto M, Yuda A, Hasegawa S, Sawada Y, et al. Possible roles of angiotensin II-forming enzymes, angiotensin converting enzyme and chymase-like enzyme, in the human aneurysmal aorta. Hypertens Res. 2002;25:817-22. 
15. Eagleton MJ, Ballard N, Lynch E, Srivastava SD, Upchurch GR Jr, Stanley JC. Early increased MT1-MMP expression and late MMP-2 and MMP-9 activity during angiotensin II induced aneurysm formation. J Surg Res. 2006;135:345-51.

16. Gordon KJ, Blobe GC. Role of transforming growth factor- $\beta$ superfamily signaling pathways in human disease. Biochim Biophys Acta. 2008;1782:197-228.

17. Loeys B, De Paepe A. New insights in the pathogenesis of aortic aneurysms. Verh K Acad Geneeskd Belg. 2008;70:69-84.

18. Heeneman S, Sluimer JC, Daemen MJ. Angiotensin-converting enzyme and vascular remodeling. Circ Res. 2007;101:441-54.

19. Nishimoto M, Takai S, Fukumoto H, Tsunemi K, Yuda A, Sawada Y. Increased local angiotensin II formation in aneurysmal aorta. Life Sci. 2002;71:2195-205.

20. Rigat B, Hubert C, Alhenc-Gelas F, Cambien F, Corvol P, Soubrier F. An insertion/ deletion polymorphism in the angiotensin I-converting enzyme gene accounting for half the variance of serum enzyme levels. J Clin Invest. 1990;86:1343-6.

21. Danser AH, Schalekamp MA, Bax WA, van den Brink AM, Saxena PR, Riegger GA. Angiotensin-converting enzyme in the human heart: effect of the deletion/insertion polymorphism. Circulation. 1995;92:1387-8.

22. Jones GT, Thompson AR, van Bockxmeer FM, Hafez H, Cooper JA, Golledge J, et al. Angiotensin II type 1 receptor $1166 \mathrm{C}$ polymorphism is associated with abdominal aortic aneurysm in three independent cohorts. Arterioscler Thromb Vasc Biol. 2008;28:764-70.

23. Bonow RO, Carabello BA, Chatterjee K, de Leon AC Jr, Faxon DP, Freed MD, et al. 2008 Focused update incorporated into the ACC/AHA 2006 guidelines for the management of patients with valvular heart disease: a report of the American College of Cardiology/American Heart Association Task Force on Practice Guidelines (Writing Committee to revise the 1998 guidelines for the management of patients with valvular heart disease). Endorsed by the Society of Cardiovascular Anesthesiologists, Society for Cardiovascular Angiography and Interventions, and Society of Thoracic Surgeons. J Am Coll Cardiol. 2008;52:e1-142.

24. Milewicz DM, Regalado ES, Guo DC. Treatment guidelines for thoracic aortic aneurysms and dissections based on the underlying causative gene. J Thorac Cardiovasc Surg. 2010;140:S2-4.

25. Sundt TM III. Replacement of the ascending aorta in bicuspid aortic valve disease: where do we draw the line? J Thorac Cardiovasc Surg. 2010;140:S41-4.

26. RoOsborne-Pellegrin MJ, Coutard M, Poitevin P, Michel JB, Levy BI. Induction of aneurysms in the rat by a stenosing cotton ligature around the inter-renal aorta. Int J Exp Pathol. 1994;75:179-90.
27. Liao S, Miralles M, Kelley BJ, Curci JA, Borhani M, Thompson RW. Suppression of experimental abdominal aortic aneurysms in the rat by treatment with angiotensin-converting enzyme inhibitors. J Vasc Surg. 2001;33:1057-64

28. Nagashima H, Uto K, Sakomura Y, Aoka Y, Sakuta A, Aomi S, et al. An angiotensin-converting enzyme inhibitor, perindopril, but not carvedilol prevents against beta-aminopropionitril monofumarate-induced aortic dissection in rat model. J Vasc Surg. 2002;36:818-23.

29. Habashi JP, Doyle JJ, Holm TM, Aziz H, Schoenhoff F, Bedja D, et al. Angiotensin II type 2 receptor signaling attenuates aortic aneurysm in mice through ERK antagonism. Science. 2011;332:361-5.

30. Hackam DG, Thiruchelvam D, Redelmeier DA. Angiotensin-converting enzyme inhibitors and aortic rupture: a population-based case-control study. Lancet. 2006;368:659-66.

31. Yetman AT, Bornemeier RA, McCrindle BW. Usefulness of enalapril versus propranolol or atenolol for prevention of aortic dilation in patients with the Marfan syndrome. Am J Cardiol. 2005;95:1125-7.

32. Ahimastos AA, Aggarwal A, D'Orsa KM, Formosa MF, White AJ, Savarirayan R, et al. Effect of perindopril on large artery stiffness and aortic root diameter in patients with Marfan syndrome: a randomized controlled trial. JAMA. 2007;298:1539-47.

33. Brooke BS, Habashi JP, Judge DP, Patel N, Loeys B, Dietz HC III. Angiotensin II blockade and aortic-root dilation in Marfan's syndrome. N Engl J Med. 2008; 358:2787-95.

34. Lacro RV, Dietz HC, Wruck LM, Bradley TJ, Colan SD, Devereux RB, et al. Rationale and design of a randomized clinical trial of beta-blocker therapy (atenolol) versus angiotensin II receptor blocker therapy (losartan) in individuals with Marfan syndrome. Am Heart J. 2007;154:624-31.

35. Radonic T, de Witte P, Baars MJ, Zwinderman AH, Mulder BJ, Groenink M, COMPARE Study Group. Losartan therapy in adults with Marfan syndrome: study protocol of the multi-center randomized controlled COMPARE trial. Trials. 2010;11:3.

36. Detaint D, Aegerter P, Tubach F, Hoffman I, Plauchu H, Dulac Y, et al. Rationale and design of a randomized clinical trial (Marfan Sartan) of angiotensin II receptor blocker therapy versus placebo in individuals with Marfan syndrome. Arch Cardiovasc Dis. 2010;103:317-25.

37. Jeunemaitre X. Genetics of the human renin angiotensin system. J Mol Med. 2008;86:637-41. 